\title{
Astro2020 Science White Paper Dark Matter Science in the Era of LSST
}

\author{
Thematic Areas: $\quad \square$ Planetary Systems \\ $\square$ Star and Planet Formation \\ Formation and Evolution of Compact Objects \\ Cosmology and Fundamental Physics \\ $\square$ Stars and Stellar Evolution $\square$ Resolved Stellar Populations and their Environments \\ $\square$ Galaxy Evolution $\quad \square$ Multi-Messenger Astronomy and Astrophysics
}

Principal Authors: Keith Bechtol ${ }^{1}$, kbechtol@wisc.edu (UW Madison) Alex Drlica-Wagner ${ }^{2,3,4}$, kadrlica@fnal.gov (Fermilab/KICP/UChicago)

Co-authors (affiliations after text): Kevork N. Abazajian ${ }^{5}$, Muntazir Abidi ${ }^{6}$, Susmita Adhikari ${ }^{7}$, Yacine Ali-Haïmoud ${ }^{8}$, James Annis ${ }^{2}$, Behzad Ansarinejad ${ }^{9}$, Robert Armstrong ${ }^{10}$, Jacobo Asorey ${ }^{11}$, Carlo Baccigalupi $^{12,13,14}$, Arka Banerjee ${ }^{7,15}$, Nilanjan Banik ${ }^{16,17}$, Charles Bennett ${ }^{18}$, Florian Beutler ${ }^{19}$, Simeon Bird ${ }^{20}$, Simon Birrer ${ }^{21}$, Rahul Biswas ${ }^{22}$, Andrea Biviano ${ }^{23}$, Jonathan Blazek ${ }^{24}$, Kimberly K. Boddy ${ }^{18}$, Ana Bonaca ${ }^{25}$, Julian Borrill ${ }^{26}$, Sownak Bose ${ }^{25}$, Jo Bovy ${ }^{27}$, Brenda Frye ${ }^{28}$, Alyson M. Brooks ${ }^{29}$, Matthew R. Buckley ${ }^{29}$, Elizabeth Buckley-Geer ${ }^{2}$, Esra Bulbul ${ }^{25}$, Patricia R. Burchat ${ }^{30}$, Cliff Burgess ${ }^{31}$, Francesca Calore ${ }^{32}$, Regina Caputo ${ }^{33}$, Emanuele Castorina ${ }^{34}$, Chihway Chang ${ }^{4,3}$, George Chapline ${ }^{10}$, Eric Charles ${ }^{7,15}$, Xingang Chen ${ }^{25}$, Douglas Clowe ${ }^{35}$, Johann Cohen-Tanugi ${ }^{36}$, Johan Comparat ${ }^{37}$, Rupert A. C. Croft ${ }^{38}$, Alessandro Cuoco ${ }^{39,40}$, Francis-Yan Cyr-Racine ${ }^{41,42}$, Guido D'Amico ${ }^{30}$, Tamara M Davis ${ }^{43,43}$, William A. Dawson ${ }^{10}$, Axel de la Macorra $^{44}$, Eleonora Di Valentino ${ }^{45}$, Ana Díaz Rivero ${ }^{41}$, Seth Digel ${ }^{7,15}$, Scott Dodelson ${ }^{38}$, Olivier Doré ${ }^{46}$, Cora Dvorkin $^{41}$, Christopher Eckner ${ }^{47}$, John Ellison ${ }^{20}$, Denis Erkal ${ }^{48}$, Arya Farahi ${ }^{38}$,

Christopher D. Fassnacht ${ }^{49}$, Pedro G. Ferreira ${ }^{50}$, Brenna Flaugher ${ }^{2}$, Simon Foreman ${ }^{51}$, Oliver Friedrich ${ }^{52}$, Joshua Frieman $^{2,3}$, Juan García-Bellido ${ }^{53}$, Eric Gawiser ${ }^{29}$, Martina Gerbino ${ }^{54}$, Maurizio Giannotti ${ }^{55}$, Mandeep S.S. Gill ${ }^{7,30,15}$, Vera Gluscevic ${ }^{56,76}$, Nathan Golovich ${ }^{10}$, Satya Gontcho A Gontcho ${ }^{57}$, Alma X. González-Morales $^{58}$, Daniel Grin ${ }^{59}$, Daniel Gruen ${ }^{7,30}$, Andrew P. Hearin ${ }^{54}$, David Hendel ${ }^{27}$, Yashar D. Hezaveh ${ }^{60}$, Christopher M. Hirata ${ }^{61}$, Renee Hložek ${ }^{27,62}$, Shunsaku Horiuchi ${ }^{63}$, Bhuvnesh Jain ${ }^{64}$, M. James Jee ${ }^{49,65}$, Tesla E. Jeltema ${ }^{66}$, Marc Kamionkowski ${ }^{18}$, Manoj Kaplinghat ${ }^{5}$, Ryan E. Keeley ${ }^{11}$, Charles R. Keeton ${ }^{29}$, Rishi Khatri ${ }^{67}$, Sergey E. Koposov ${ }^{38,68}$, Savvas M. Koushiappas ${ }^{69}$, Ely D. Kovetz ${ }^{70}$, Ofer Lahav ${ }^{71}$, Casey Lam ${ }^{72}$, Chien-Hsiu Lee ${ }^{73}$, Ting S. Li ${ }^{2,3}$, Michele Liguori ${ }^{74}$, Tongyan Lin ${ }^{75}$, Mariangela Lisanti ${ }^{76}$, Marilena LoVerde ${ }^{77}$, Jessica R. Lu ${ }^{72}$, Rachel Mandelbaum ${ }^{38}$, Yao-Yuan Mao ${ }^{78}$, Samuel D. McDermott ${ }^{2}$, Mitch McNanna ${ }^{1}$, Michael Medford ${ }^{72,26}$, P. Daniel Meerburg ${ }^{52,6,79}$, Manuel Meyer ${ }^{7,15}$, Mehrdad Mirbabayi ${ }^{80}$, Siddharth Mishra-Sharma ${ }^{8}$, Moniez Marc ${ }^{81}$, Surhud More ${ }^{82}$, John Moustakas $^{83}$, Julian B. Muñoz ${ }^{41}$, Simona Murgia ${ }^{5}$, Adam D. Myers ${ }^{84}$, Ethan O. Nadler ${ }^{7,30}$, Lina Necib ${ }^{85}$, Laura Newburgh ${ }^{86}$, Jeffrey A. Newman ${ }^{78}$, Brian Nord ${ }^{2,3,4}$, Erfan Nourbakhsh ${ }^{49}$, Eric Nuss ${ }^{36}$, Paul O'Connor ${ }^{87}$, Andrew B. Pace ${ }^{88}$, Hamsa Padmanabhan ${ }^{51,89}$, Antonella Palmese ${ }^{2}$, Hiranya V. Peiris $^{71,22}$, Annika H. G. Peter ${ }^{61,90,91}$, Francesco Piacentni ${ }^{92,93}$, Andrés Plazas ${ }^{76}$, Daniel A. Polin ${ }^{49}$, Abhishek Prakash $^{85}$, Chanda Prescod-Weinstein ${ }^{94}$, Justin I. Read ${ }^{48}$, Steven Ritz ${ }^{66}$, Brant E. Robertson ${ }^{66}$, Benjamin Rose ${ }^{95}$, Rogerio Rosenfeld ${ }^{80,96}$, Graziano Rossi ${ }^{97}$, Lado Samushia ${ }^{98}$, Javier Sánchez ${ }^{5}$, Miguel A. Sánchez-Conde ${ }^{53,99}$, Emmanuel Schaan ${ }^{26,34}$, Neelima Sehgal ${ }^{100}$, Leonardo Senatore ${ }^{7}$, Hee-Jong $\mathrm{Seo}^{35}$, Arman Shafieloo ${ }^{11}$, Huanyuan Shan ${ }^{101}$, Nora Shipp ${ }^{4}$, Joshua D. Simon ${ }^{102}$, Sara Simon ${ }^{103}$, Tracy R. Slatyer ${ }^{104}$, Anže Slosar ${ }^{87}$, Srivatsan Sridhar ${ }^{11}$, Albert Stebbins ${ }^{2}$, Oscar Straniero ${ }^{105}$, Louis E. Strigari ${ }^{88}$, Tim M. P. Tait ${ }^{5}$, Erik Tollerud ${ }^{106}$, M. A. Troxel ${ }^{107,108}$, J. Anthony Tyson ${ }^{49}$, Cora Uhlemann $^{6}$, L. Arturo Urenña-López ${ }^{109}$, Aprajita Verma ${ }^{50}$, Ricardo Vilalta ${ }^{110}$, Christopher W. Walter ${ }^{108}$, Mei-Yu Wang ${ }^{38}$, Scott Watson ${ }^{111}$, Risa H. Wechsler ${ }^{7,30,15}$, David Wittman ${ }^{49}$, Weishuang $\mathrm{Xu}^{41}$, 
Brian Yanny ${ }^{2}$, Sam Young ${ }^{112}$, Hai-Bo Yu ${ }^{20}$, Gabrijela Zaharijas ${ }^{113}$, Andrew R. Zentner ${ }^{78}$, Joe Zuntz ${ }^{114}$

Abstract: Astrophysical observations currently provide the only robust, empirical measurements of dark matter. In the coming decade, astrophysical observations will guide other experimental efforts, while simultaneously probing unique regions of dark matter parameter space. This white paper summarizes astrophysical observations that can constrain the fundamental physics of dark matter in the era of LSST. We describe how astrophysical observations will inform our understanding of the fundamental properties of dark matter, such as particle mass, self-interaction strength, non-gravitational interactions with the Standard Model, and compact object abundances. Additionally, we highlight theoretical work and experimental/observational facilities that will complement LSST to strengthen our understanding of the fundamental characteristics of dark matter. 


\section{Summary}

More than 85 years after its astrophysical discovery, the fundamental nature of dark matter remains one of the foremost open questions in science. Over the last several decades, an extensive experimental program has sought to determine the cosmological origin, constituents, and interaction mechanisms of dark matter. To date, the only direct, positive empirical measurements of dark matter come from astrophysical observations. Discovering the fundamental nature of dark matter will necessarily draw upon the tools particle physics, cosmology, and astronomy.

LSST will provide a unique and impressive platform to study dark sector physics in the 2020s. Originally envisioned as the "Dark Matter Telescope" ${ }^{1}$, LSST will enable precision tests of the $\Lambda \mathrm{CDM}$ model and elucidate the connection between luminous galaxies and the cosmic web of dark matter. Cosmology has consistently shown that it is impossible to separate the macroscopic distribution of dark matter from the microscopic physics governing dark matter. In fact, some microscopic characteristics of dark matter are only accessible via astrophysics. Studies of dark matter, dark energy, massive neutrinos, and galaxy evolution are extremely complementary from both a technical and scientific standpoint. A robust dark matter program leveraging LSST data has the ability to test a broad range of well-motivated theoretical models of dark matter including selfinteracting dark matter, warm dark matter, dark matter-baryon scattering, ultra-light dark matter, axion-like particles, and primordial black holes.

LSST will enable studies of Milky Way satellite galaxies, stellar streams, and strong lens systems to detect and characterize the smallest dark matter halos, thereby probing the minimum mass of ultra-light dark matter and thermal warm dark matter. Precise measurements of the density and shapes of dark matter halos in dwarf galaxies and galaxy clusters will be sensitive to dark matter self-interactions probing hidden sector and dark photon models. Microlensing measurements will directly probe primordial black holes and the compact object fraction of dark matter at the sub-percent level over a wide range of masses. Precise measurements of stellar populations will be sensitive to anomalous energy loss mechanisms and will constrain the coupling of axion-like particles to photons and electrons. Measurements of large-scale structure will spatially resolve the influence of both dark matter and dark energy, enabling searches for correlations between the two known components of the dark sector. In addition, complementarity between astrophysical, direct detection, and other indirect searches for dark matter will help constrain dark matter-baryon scattering, dark matter self-annihilation, and dark matter decay.

Astrophysical dark matter studies will explore parameter space beyond the current sensitivity of the high-energy physics program and will complement other experimental searches. This has been recognized in Astro $2010^{2}$, during the Snowmass Cosmic Frontier planning process ${ }^{3-5}$, in the P5 Report ${ }^{6}$, and in a series of recent Cosmic Visions reports ${ }^{7 ; 8}$, including the "New Ideas in Dark Matter 2017: Community Report" 9 . In the 2020s, the impact of the LSST dark matter program will be enhanced by access to wide-field massively multiplexed spectroscopy on medium- to largeaperture telescopes ( $\sim 8-10$-meter class), deep spectroscopy on giant segmented mirror telescopes ( 30-m class), together with high-resolution optical and radio imaging. Further theoretical work is also needed to interpret those observations in terms of particle models, to combine results from multiple observational methods, and to develop novel probes of dark matter.

This whitepaper is a summary of Drlica-Wagner et al. (2019) ${ }^{10}$. 


\begin{tabular}{lccc}
\hline Model & Probe & Parameter & Value \\
\hline \hline Warm Dark Matter & Halo Mass & Particle Mass & $m \sim 18 \mathrm{keV}$ \\
Self-Interacting Dark Matter & Halo Profile & Cross Section & $\sigma_{\text {SIDM }} / m_{\chi} \sim 0.1-10 \mathrm{~cm}^{2} / \mathrm{g}$ \\
Baryon-Scattering Dark Matter & Halo Mass & Cross Section & $\sigma \sim 10^{-30} \mathrm{~cm}^{2}$ \\
Axion-Like Particles & Energy Loss & Coupling Strength & $g_{\phi e} \sim 10^{-13}$ \\
Fuzzy Dark Matter & Halo Mass & Particle Mass & $m \sim 10^{-20} \mathrm{eV}$ \\
Primordial Black Holes & Compact Objects & Object Mass & $M>10^{-4} M_{\odot}$ \\
WIMPs & Indirect Detection & Cross Section & $\langle\sigma v\rangle \sim 10^{-27} \mathrm{~cm}^{3} / \mathrm{s}$ \\
Light Relics & Large-Scale Structure & Relativistic Species & $N_{\text {eff }} \sim 0.1$ \\
& & &
\end{tabular}

Table 1: Probes of fundamental dark matter physics in the LSST era, organized by dark matter model and associated observables. Sensitivity forecasts appear in the rightmost column.

\section{Dark Matter Models}

Astrophysical observations probe the physics of dark matter through its impact on structure formation throughout cosmic history. On large scales, current observational data are well described by a simple model of stable, non-relativistic, collisionless, cold dark matter (CDM). However, many viable theoretical models of dark matter predict deviations from CDM that are testable with current and future observations. Fundamental properties of dark matter-e.g., particle mass, selfinteraction cross section, coupling to the Standard Model, and time evolution-can imprint themselves on the macroscopic distribution of dark matter in a detectable manner. With supporting theoretical efforts and follow-up observations, LSST will be sensitive to several distinct classes of dark matter models, including particle dark matter, field dark matter, and compact objects (Table 1).

Particle Dark Matter: LSST, in combination with other observations, will be able to probe microscopic characteristics of particle dark matter such as self-interaction cross section, particle mass, baryon-scattering cross section, self-annihilation rate, and decay rate. These measurements will complement and guide collider, direct, and indirect detection efforts to study particle dark matter.

Wave-like Dark Matter: Axion-like particles and other (ultra-)light dark matter candidates are a natural alternative to conventional particle dark matter. LSST will be uniquely sensitive to the minimum mass of ultra-light dark matter and to couplings between axion-like particles and the Standard Model.

Compact Objects: Compact object dark matter is fundamentally different from particle models; primordial black holes cannot be studied in an accelerator and can only be detected through their gravitational force. Primordial black holes (PBHs) formed directly from the primordial density fluctuations could make up some fraction of the dark matter, and a measurement of their abundance would directly constrain the amplitude of density fluctuations and provide unique insights into physics at ultra-high energies.

\section{Dark Matter Probes}

Minimum Halo Mass: The standard cosmological model predicts a nearly scale-invariant mass spectrum of dark matter halos down to Earth-mass scales (or below), e.g., in WIMP and nonthermal axion models ${ }^{11-13}$. Modifications to the cold, collisionless dark matter paradigm can suppress the formation of dark matter halos on these small scales. Current observations provide a 
robust measurement of the dark matter halo mass spectrum for halos with mass $>10^{10} M_{\odot}$, and the smallest known galaxies provide an existence proof for halos of mass $\sim 10^{8} M_{\odot}-10^{9} M_{\odot}{ }^{14-19}$. LSST will expand the census of ultra-faint satellite galaxies orbiting the Milky Way and enable statistical searches for extremely low-luminosity and low-surface brightness galaxies throughout the Local Volume. By measuring the galaxy luminosity function at the extreme low-mass threshold of galaxy formation, LSST will test the abundance of dark matter halos at $\sim 10^{8} M_{\odot}$.

LSST will probe dark matter halos below the threshold of galaxy formation with stellar streams and strongly lensed systems. Galactic dark matter subhalos with masses as small as $10^{5}-10^{6} M_{\odot}$ passing a stellar stream are capable of producing detectable gaps in the stellar density ${ }^{20 ; 21}$. By identifying additional stellar streams and increasing the density contrast of known streams against the smooth Milky Way halo, LSST will shift analysis from individual gaps into the regime of subhalo population statistics and (in)consistency with cold dark matter predictions. Importantly, LSST will allow studies of streams farther from the center of the Galaxy for which confounding baryonic effects are lessened. Meanwhile, strong gravitational lensing can be used to measure the abundance and masses of subhalos in massive galaxies and small isolated halos along the line of sight at cosmological distances, independent of their baryon content. LSST will increase the number of lensed systems from the current sample of hundreds to an expected sample of thousands of lensed quasars ${ }^{22}$ and tens of thousands of lensed galaxies ${ }^{23}$.

Halo Profiles: Measurements of the radial density profiles and shapes of dark matter halos are sensitive to the microphysics governing non-gravitational dark matter self-interactions, which could produce flat density cores ${ }^{24}$ and more spherical halo shapes ${ }^{25}$. Through galaxy-galaxy weak lensing, LSST will be able to distinguish cored versus cuspy NFW density profiles for a sample of low-redshift dwarf galaxies with masses $M_{\text {halo }}=3 \times 10^{9} h^{-1} M_{\odot}$. Studies of the density profiles of massive galaxy clusters, as well as systems of merging galaxy clusters, will constrain the scattering cross section at the level $\sigma_{\text {SIDM }} / m_{\chi} \sim 0.1-1 \mathrm{~cm}^{2} \mathrm{~g}^{-1}$. Measuring halo profiles over a range of mass scales will provide sensitivity to dark matter scattering with non-trivial velocity dependence.

Compact Objects: LSST has the ability to directly detect signals of compact halo objects through precise, short- $(\sim 30 \mathrm{~s})$ and long-duration $(\sim 1 \mathrm{yr})$ observations of classical and parallactic microlensing ${ }^{26}$. If scheduled optimally, LSST could extend PBH sensitivity to $\sim 0.03 \%$ of the dark matter fraction for masses $\gtrsim 10^{-1} M_{\odot}$. By supplementing the LSST survey with astrometric microlensing observations, it will be possible to break lensing mass-geometry degeneracies and make precise measurements of individual black hole masses. Thus, if PBHs make up a significant fraction of dark matter, LSST will effectively measure their "particle" properties and provide insight into the fundamental physics of the early universe.

Anomalous Energy Loss: Observations of stars provide a mechanism to probe temperatures, particle densities, and time scales that are inaccessible to laboratory experiments. Since conventional astrophysics allows us to quantitatively model the evolution of stars, detailed study of stellar populations can provide a powerful technique to probe new physics. In particular, if new light particles exist and are coupled to Standard Model fields, their emission would provide an additional channel for stellar energy loss. LSST will greatly improve our understanding of stellar evolution by providing unprecedented photometry, astrometry, and temporal sampling for a large sample of faint stars. In particular, measurements of the white dwarf luminosity function, giant branch stars, and core-collapse supernovae will provide sensitivity to the axion-electron coupling. 

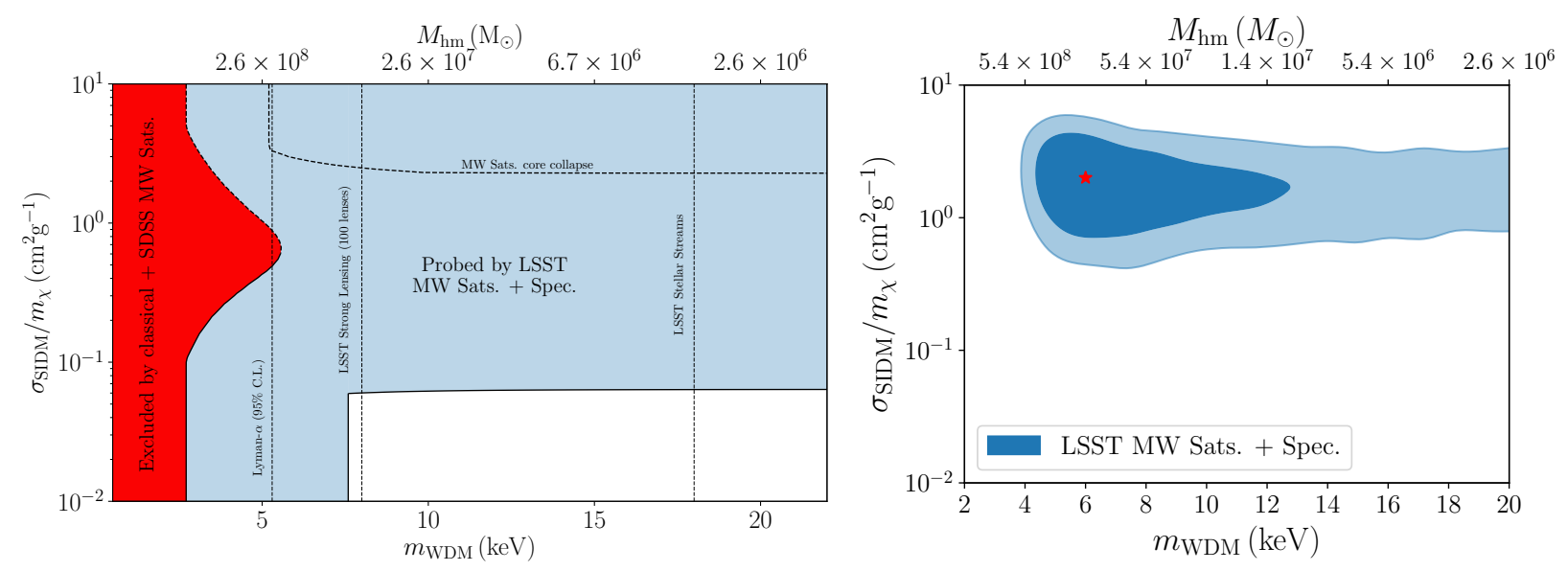

Figure 1: Left: Projected joint sensitivity to WDM particle mass and SIDM cross section from LSST observations of dark matter substructure. Right: Example of a measurement of particle properties for a dark matter model with a self-interaction cross section and matter power spectrum cut-off just beyond current constraints $\left(\sigma_{\mathrm{SIDM}} / m_{\chi}=2 \mathrm{~cm}^{2} \mathrm{~g}^{-1}\right.$ and $m_{\mathrm{WDM}}=6 \mathrm{keV}$, indicated by the red star $)^{10}$. Complementary observations can break degeneracies among dark matter models that have the same approximate behavior on small scales but differ in detail.

Large-Scale Structure: LSST will produce the largest and most detailed map of the distribution of matter and the growth of cosmic structure over the past 10 Gyr. The large-scale clustering of matter and luminous tracers in the late-time universe is sensitive to the total amount of dark matter, the fraction of dark matter in light relics that behave as radiation at early times, and fundamental couplings between dark matter and dark energy. Measurements of large-scale structure with LSST will enhance constraints on massive neutrinos and other light relics from the early universe that could compose a fraction of the dark matter. Additionally, LSST will use supernovae and $3 \times 2 \mathrm{pt}$ statistics of galaxy clustering and weak lensing to measure dark energy in independent patches across the sky, allowing for spatial cross correlation between dark matter and dark energy ${ }^{27}$.

\section{Complementarity}

LSST will enable complementary studies of dark matter with spectroscopy, high-resolution imaging, indirect detection experiments, and direct detection experiments. While LSST can substantially improve our understanding of dark matter in isolation, the combination of experiments is essential to confirm future discoveries and provide a holistic picture of dark matter physics.

Spectroscopy: Wide field-of-view, massively multiplexed spectroscopy on 8-10-meter-class telescopes as well as deep spectroscopy with 30-meter-class telescopes will complement studies of minimum halo mass and halo profiles.

High-Resolution Imaging: High-resolution follow-up imaging at the milliarcsecond-scale from space and with ground-based adaptive optics are needed to maximize strong lensing, microlensing, and galaxy cluster studies with LSST.

Indirect Detection: By precisely mapping the distribution of dark matter on Galactic and extragalactic scales, LSST will enable more sensitive searches for energetic particles created by dark 

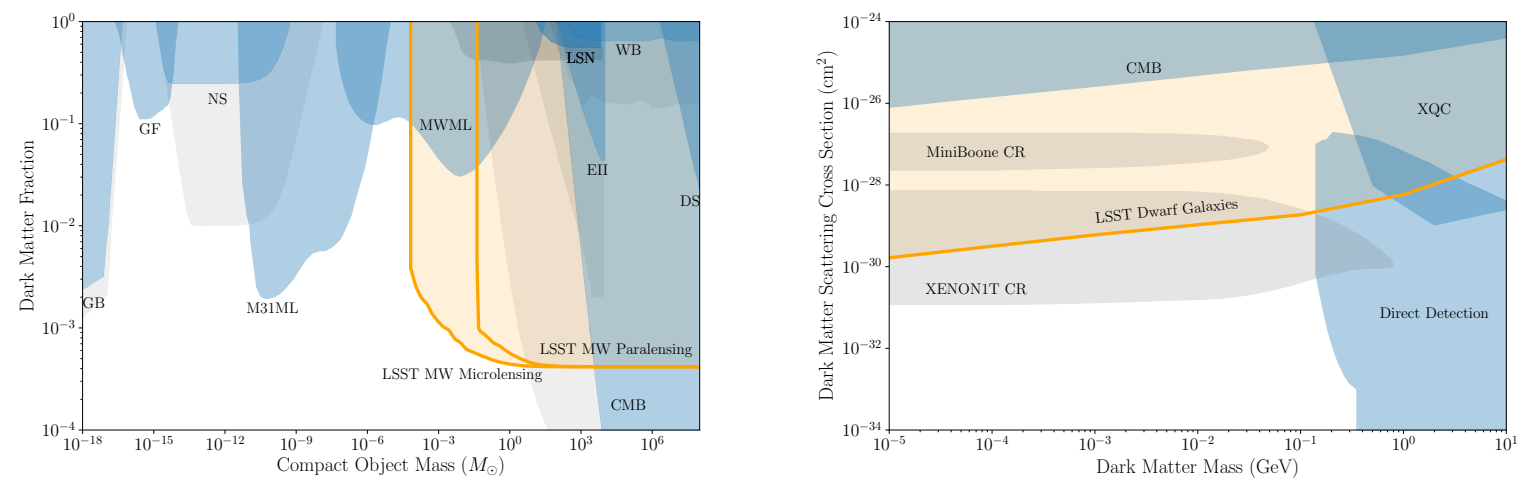

Figure 2: Left: Constraints on the maximal fraction of dark matter in compact objects from existing probes (blue and gray) and projected sensitivity for LSST microlensing measurements (gold). Right: Constraints on dark matter-baryon scattering through a velocity-independent, spinindependent contact interaction with protons from existing constraints (blue and gray) and projections for LSST observations of Milky Way satellite galaxies (gold) ${ }^{10 ; 32}$.

matter annihilation and/or decay, e.g., using gamma-ray or neutrino telescopes ${ }^{28-30}$. LSST will also provide sensitivity to axion-like particles via monitoring extreme events in the transient sky ${ }^{31}$.

Direct Detection: LSST will complement direct detection experiments by improving measurements of the local phase-space density of dark matter using precision astrometry of Milky Way stars. For dark matter-baryon scattering, small-scale structure measurements with LSST can probe dark matter masses and cross sections outside the range accessible to direct detection experiments.

\section{Recommendations for Astro 2020}

LSST is scheduled to begin a decade of science operation in 2022; however, dark matter research with LSST is not yet funded. Recognizing new opportunities created by LSST to constrain a range of dark matter models, we make the following recommendations to facilitate this science case:

- Support individual PIs and collaborative teams to analyze LSST data for dark matter science.

- Support associated theoretical research to better understand the galaxy-halo connection, examine confounding baryonic effects, perform joint analyses of cosmological probes, investigate novel signatures of dark matter microphysics, and strengthen ties with the particle physics community.

- Support complementary observational facilities to investigate dark matter, including spectroscopic follow-up and high-resolution imaging, as well as multiwavelength analyses.

We anticipate that the multi-faceted LSST data will allow further probes of dark matter physics that have yet to be considered. New ideas are especially important as searches for the most popular dark matter candidates gain in sensitivity while lacking a positive detection. As the particle physics community seeks to diversify the experimental effort to search for dark matter, it is important to remember that astrophysical observations provide robust, empirical measurement of fundamental dark matter properties. In the coming decade, astrophysical observations will guide other experimental efforts, while simultaneously probing unique regions of dark matter parameter space. 


\section{References}

[1] J. A. Tyson, D. M. Wittman and J. R. P. Angel, The Dark Matter Telescope, in Gravitational Lensing: Recent Progress and Future Goals, T. G. Brainerd and C. S. Kochanek, eds., vol. 237 of Astronomical Society of the Pacific Conference Series, p. 417, January, 2001, a stro-ph/ 0005381.

[2] National Research Council, New Worlds, New Horizons in Astronomy and Astrophysics. National Academies Press, 2010, 10.17226/12951.

[3] A. Kusenko and L. J. Rosenberg, Snowmass-2013 Cosmic Frontier 3 (CF3) Working Group Summary: Non-WIMP dark matter, arXiv e-prints (2013) arXiv:1310.8642 [1310 . 8642].

[4] J. J. Beatty, A. E. Nelson, A. Olinto, G. Sinnis, A. U. Abeysekara, L. A. Anchordoqui et al., Snowmass Cosmic Frontiers 6 (CF6) Working Group Summary -The Bright Side of the Cosmic Frontier: Cosmic Probes of Fundamental Physics, arXiv e-prints (2013) arXiv:1310.5662 [1310.5662].

[5] D. Bauer, J. Buckley, M. Cahill-Rowley, R. Cotta, A. Drlica-Wagner, J. L. Feng et al., Dark matter in the coming decade: Complementary paths to discovery and beyond, Physics of the Dark Universe 7 (2015) 16 [1305.1605].

[6] S. Ritz, H. Aihara, M. Breidenbach, B. Cousins, A. de Gouvea, M. Demarteau et al., Building for Discovery: Strategic Plan for U.S. Particle Physics in the Global Context. HEPAP Subcommittee, 2014.

[7] S. Dodelson, K. Heitmann, C. Hirata, K. Honscheid, A. Roodman, U. Seljak et al., Cosmic Visions Dark Energy: Science, arXiv e-prints (2016) arXiv:1604.07626 [1604.07626].

[8] K. Dawson, J. Frieman, K. Heitmann, B. Jain, S. Kahn, R. Mandelbaum et al., Cosmic Visions Dark Energy: Small Projects Portfolio, arXiv e-prints (2018) arXiv:1802.07216 [1802 . 07216].

[9] M. Battaglieri, A. Belloni, A. Chou, P. Cushman, B. Echenard, R. Essig et al., US Cosmic Visions: New Ideas in Dark Matter 2017: Community Report, arXiv e-prints (2017) arXiv:1707.04591 [1707.04591].

[10] A. Drlica-Wagner, Y.-Y. Mao, S. Adhikari, R. Armstrong, A. Banerjee, N. Banik et al., Probing the Fundamental Nature of Dark Matter with the Large Synoptic Survey Telescope, arXiv e-prints (2019) arXiv:1902.01055 [1902.01055].

[11] A. M. Green, S. Hofmann and D. J. Schwarz, The power spectrum of SUSY-CDM on subgalactic scales, MNRAS 353 (2004) L23 [astro-ph / 0309621 ].

[12] J. Diemand, B. Moore and J. Stadel, Earth-mass dark-matter haloes as the first structures in the early Universe, Nature 433 (2005) 389 [ast ro-ph/ 0501589 ].

[13] A. H. Guth, M. P. Hertzberg and C. Prescod-Weinstein, Do dark matter axions form a condensate with long-range correlation?, Phys. Rev. D 92 (2015) 103513 [1412. 5930].

[14] J. I. Read, G. Iorio, O. Agertz and F. Fraternali, The stellar mass-halo mass relation of isolated field dwarfs: a critical test of $\Lambda C D M$ at the edge of galaxy formation, MNRAS 467 (2017) 2019 [1607.03127].

[15] P. Behroozi, R. Wechsler, A. Hearin and C. Conroy, UniverseMachine: The Correlation between Galaxy Growth and Dark Matter Halo Assembly from $z=0-10$, arXiv e-prints (2018) arXiv:1806.07893 [1806.07893].

[16] P. Jethwa, D. Erkal and V. Belokurov, The upper bound on the lowest mass halo, MNRAS 473 (2018) $2060[1612.07834]$.

[17] S. Y. Kim, A. H. G. Peter and J. R. Hargis, Missing Satellites Problem: Completeness Corrections to the Number of Satellite Galaxies in the Milky Way are Consistent with Cold Dark Matter Predictions, Phys. Rev. Lett. 121 (2018) 211302. 
[18] E. O. Nadler, Y.-Y. Mao, G. M. Green and R. H. Wechsler, Modeling the Connection Between Subhalos and Satellites in Milky Way-Like Systems, arXiv e-prints (2018) arXiv:1809.05542 [1809.05542].

[19] J. I. Read and D. Erkal, Abundance matching with the mean star formation rate: there is no missing satellites problem in the Milky Way, arXiv e-prints (2018) arXiv:1807.07093 [1807.07093].

[20] D. Erkal, V. Belokurov, J. Bovy and J. L. Sanders, The number and size of subhalo-induced gaps in stellar streams, MNRAS 463 (2016) 102 [1606.04946].

[21] J. Bovy, D. Erkal and J. L. Sanders, Linear perturbation theory for tidal streams and the small-scale CDM power spectrum, MNRAS 466 (2017) 628 [1606.03470].

[22] M. Oguri and P. J. Marshall, Gravitationally lensed quasars and supernovae in future wide-field optical imaging surveys, MNRAS 405 (2010) 2579 [1001.2037].

[23] T. E. Collett, The Population of Galaxy-Galaxy Strong Lenses in Forthcoming Optical Imaging Surveys, ApJ 811 (2015) 20 [1507.02657].

[24] D. N. Spergel and P. J. Steinhardt, Observational Evidence for Self-Interacting Cold Dark Matter, Phys. Rev. Lett. 84 (2000) 3760 [astro-ph/9909386].

[25] A. H. G. Peter, M. Rocha, J. S. Bullock and M. Kaplinghat, Cosmological simulations with self-interacting dark matter - II. Halo shapes versus observations, MNRAS 430 (2013) 105 [1208.3026].

[26] Ł. Wyrzykowski, Z. Kostrzewa-Rutkowska, J. Skowron, K. A. Rybicki, P. Mróz, S. Kozłowski et al., Black hole, neutron star and white dwarf candidates from microlensing with OGLE-III, MNRAS 458 (2016) 3012 [1509.04899].

[27] R. Scranton, A. Albrecht, R. Caldwell, A. Cooray, O. Dore, S. Habib et al., The Case for Deep, Wide-Field Cosmology, in astro2010: The Astronomy and Astrophysics Decadal Survey, vol. 2010, p. 269, January, 2009, 0902.2590.

[28] E. Charles, M. Sánchez-Conde, B. Anderson, R. Caputo, A. Cuoco, M. Di Mauro et al., Sensitivity projections for dark matter searches with the Fermi large area telescope, Phys. Rep. 636 (2016) 1 [1605.02016].

[29] A. Albert, B. Anderson, K. Bechtol, A. Drlica-Wagner, M. Meyer, M. Sánchez-Conde et al., Searching for Dark Matter Annihilation in Recently Discovered Milky Way Satellites with Fermi-Lat, ApJ 834 (2017) 110 [1611.03184].

[30] M. Shirasaki, S. Horiuchi and N. Yoshida, Cross correlation of cosmic shear and extragalactic gamma-ray background: Constraints on the dark matter annihilation cross section, Phys. Rev. D 90 (2014) 063502 [1404.5503].

[31] M. Meyer, M. Giannotti, A. Mirizzi, J. Conrad and M. A. Sánchez-Conde, Fermi Large Area Telescope as a Galactic Supernovae Axionscope, Phys. Rev. Lett. 118 (2017) 011103 [1609.02350].

[32] V. Gluscevic, E. O. Nadler and K. K. Boddy (in prep). 


\section{Affiliations}

${ }^{1}$ Department of Physics, University of Wisconsin - Madison, Madison, WI 53706

${ }^{2}$ Fermi National Accelerator Laboratory, Batavia, IL 60510

${ }^{3}$ Kavli Institute for Cosmological Physics, Chicago, IL 60637

${ }^{4}$ University of Chicago, Chicago, IL 60637

${ }^{5}$ University of California, Irvine, CA 92697

${ }^{6}$ DAMTP, Centre for Mathematical Sciences, Wilberforce Road, Cambridge, UK, CB3 0WA

7 Kavli Institute for Particle Astrophysics and Cosmology, Stanford 94305

${ }^{8}$ New York University, New York, NY 10003

${ }^{9}$ Department of Physics, Lower Mountjoy, South Rd, Durham DH1 3LE, United Kingdom

${ }^{10}$ Lawrence Livermore National Laboratory, Livermore, CA, 94550

${ }^{11}$ Korea Astronomy and Space Science Institute, Daejeon 34055, Korea

12 SISSA - International School for Advanced Studies, Via Bonomea 265, 34136 Trieste, Italy

${ }^{13}$ IFPU - Institute for Fundamental Physics of the Universe, Via Beirut 2, 34014 Trieste, Italy

${ }^{14}$ INFN - National Institute for Nuclear Physics, Via Valerio 2, I-34127 Trieste, Italy

${ }^{15}$ SLAC National Accelerator Laboratory, Menlo Park, CA 94025

16 GRAPPA Institute, University of Amsterdam, Science Park 904, 1098 XH Amsterdam, The Netherlands

17 Lorentz Institute, Leiden University, Niels Bohrweg 2,Leiden, NL 2333 CA, The Netherlands

18 Johns Hopkins University, Baltimore, MD 21218

19 Institute of Cosmology \& Gravitation, University of Portsmouth, Dennis Sciama Building, Burnaby Road, Portsmouth PO1 3FX, UK

${ }^{20}$ University of California at Riverside, Riverside, CA 92521

${ }^{21}$ University of California at Los Angeles, Los Angeles, CA 90095

22 Oskar Klein Centre for Cosmoparticle Physics, Stockholm University, AlbaNova, Stockholm SE-106 91, Sweden

23 INAF - Osservatorio Astronomico di Trieste, Via G.B. Tiepolo 11, 34143 Trieste, Italy

${ }^{24}$ Institute of Physics, Laboratory of Astrophysics, Ecole Polytechnique Fédérale de Lausanne (EPFL), Observatoire de Sauverny, 1290 Versoix, Switzerland

${ }^{25}$ Harvard-Smithsonian Center for Astrophysics, MA 02138

${ }^{26}$ Lawrence Berkeley National Laboratory, Berkeley, CA 94720

${ }^{27}$ Department of Astronomy and Astrophysics, University of Toronto, $\mathrm{ON}, \mathrm{M} 5 \mathrm{~S} 3 \mathrm{H} 4$

28 Department of Astronomy/Steward Observatory, University of Arizona, Tucson, AZ 85721

${ }^{29}$ Department of Physics and Astronomy, Rutgers, the State University of New Jersey, 136 Frelinghuysen Road, Piscataway, NJ 08854, USA

${ }^{30}$ Stanford University, Stanford, CA 94305

${ }^{31}$ Perimeter Institute, Waterloo, Ontario N2L 2Y5, Canada

32 CNRS, Laboratoire d'Annecy-le-Vieux de Physique Théorique, France

${ }^{33}$ NASA Goddard Space Flight Center

${ }^{34}$ Department of Physics, University of California Berkeley, Berkeley, CA 94720, USA

35 Department of Physics and Astronomy, Ohio University, Clippinger Labs, Athens, OH 45701, USA

${ }^{36}$ Laboratoire Univers et Particules de Montpellier, Univ. Montpellier and CNRS, 34090 Montpellier, France

37 Max-Planck-Institut für extraterrestrische Physik (MPE), Giessenbachstrasse 1, D-85748 Garching bei München, Germany

${ }^{38}$ Department of Physics, McWilliams Center for Cosmology, Carnegie Mellon University

39 Institute for Theoretical Particle Physics and Cosmology, RWTH Aachen University, Germany

${ }^{40}$ Univ. Grenoble Alpes, USMB, CNRS, LAPTh, F-74940 Annecy, France
${ }^{41}$ Department of Physics, Harvard University, Cambridge, MA 02138, USA

${ }^{42}$ University of New Mexico, Albuquerque, NM 87131

43 The University of Queensland, School of Mathematics and Physics, QLD 4072, Australia

${ }^{44}$ IFUNAM - Instituto de Física, Universidad Nacional Autónoma de Mético, 04510 CDMX, México'

45 Jodrell Bank Center for Astrophysics, School of Physics and Astronomy, University of Manchester, Oxford Road, Manchester, M13 9PL, UK

46 Jet Propulsion Laboratory, California Institute of Technology, Pasadena, CA, USA

${ }^{47}$ Laboratory for Astroparticle Physics, University of Nova Gorica

${ }^{48}$ Department of Physics, University of Surrey, UK

${ }^{49}$ University of California at Davis, Davis, CA 95616

50 The University of Oxford, Oxford OX1 3RH, UK

51 Canadian Institute for Theoretical Astrophysics, University of Toronto, Toronto, ON M5S 3H8, Canada

${ }^{52}$ Kavli Institute for Cosmology, Cambridge, UK, CB3 0HA

53 Instituto de Fisica Teorica UAM/CSIC, Universidad Autonoma de Madrid, 28049 Madrid, Spain

${ }^{54}$ HEP Division, Argonne National Laboratory, Lemont, IL 60439, USA

55 Physical Science Department, Barry University

56 University of Florida, Gainesville, FL 32611

57 Department of Physics and Astronomy, University of Rochester, 500 Joseph C. Wilson Boulevard, Rochester, NY 14627, USA

58 División de Ciencias e Ingenierías, Universidad de Guanajuato, León 37150, México

${ }^{59}$ Haverford College, 370 Lancaster Ave, Haverford PA, 19041, USA

${ }^{60}$ Center for Computational Astrophysics, 162 5th Ave, 10010, New York, NY, USA

${ }^{61}$ The Ohio State University, Columbus, OH 43212

62 Dunlap Institute for Astronomy and Astrophysics, University of Toronto, ON, M5S3H4

63 Virginia Tech, Blacksburg, VA 24061

${ }^{64}$ Department of Physics and Astronomy, University of Pennsylvania, Philadelphia, Pennsylvania 19104, USA

65 Yonsei University, Seoul, South Korea

66 University of California at Santa Cruz, Santa Cruz, CA 95064

67 Tata Institute of Fundamental Research, Homi Bhabha Road, Mumbai 400005 India

${ }^{68}$ Institute of Astronomy, University of Cambridge,Cambridge CB3 OHA, UK

${ }^{69}$ Brown University, Providence, RI 02912

70 Department of Physics, Ben-Gurion University, Be'er Sheva 84105, Israel

${ }^{71}$ University College London, WC1E 6BT London, United Kingdom

72 Department of Astronomy, University of California Berkeley, Berkeley, CA 94720, USA

${ }^{73}$ National Optical Astronomy Observatory, 950 N. Cherry Ave., Tucson, AZ 85719 USA

74 Dipartimento di Fisica e Astronomia “G. Galilei”,Università degli Studi di Padova, via Marzolo 8, I-35131, Padova, Italy

75 University of California San Diego, La Jolla, CA 92093

76 Princeton University, Princeton, NJ 08544

77 C.N. Yang Institute for Theoretical Physics State University of New York Stony Brook, NY 11794

${ }^{78}$ University of Pittsburgh and PITT PACC, Pittsburgh, PA 15260

${ }^{79}$ Van Swinderen Institute for Particle Physics and Gravity, University of Groningen, Nijenborgh 4, 9747 AG Groningen, The Netherlands

${ }^{80}$ International Centre for Theoretical Physics, Strada Costiera, 11, I-34151 Trieste, Italy

${ }^{81}$ Laboratoire de l'Accélérateur Linéaire, IN2P3-CNRS, France

82 The Inter-University Centre for Astronomy and Astrophysics, Pune, 
411007, India

83 Siena College, 515 Loudon Road, Loudonville, NY 12211, USA

84 Department of Physics and Astronomy, University of Wyoming, Laramie, WY 82071, USA

85 California Institute of Technology, Pasadena, CA 91125

86 Department of Physics, Yale University, New Haven, CT 06520

87 Brookhaven National Laboratory, Upton, NY 11973

88 Texas AandM University, College Station, TX 77843

${ }^{89}$ ETH Zurich, Institute for Particle Physics, 8093 Zurich, Switzerland

90 Center for Cosmology and AstroParticle Physics, The Ohio State University

91 Department of Astronomy, The Ohio State University

92 Dipartimento di Fisica, Università La Sapienza, P. le A. Moro 2, Roma, Italy

93 Istituto Nazionale di Fisica Nucleare, Sezione di Roma, 00185 Roma, Italy

94 University of New Hampshire, Durham, NH 03824

95 Space Telescope Science Institute, Baltimore, MD 21218

96 Laboratório Interinstitucional de e-Astronomia - LIneA, Rua Gal. José Cristino 77, Rio de Janeiro, RJ - 20921-400, Brazil

97 Department of Physics and Astronomy, Sejong University, Seoul, 143-747, Korea

98 Kansas State University, Manhattan, KS 66506
99 Departamento de Física Teórica, M-15, Universidad Autónoma de Madrid, E-28049 Madrid, Spain

100 Stony Brook University, Stony Brook, NY 11794

101 Shanghai Astronomical Observatory (SHAO), Nandan Road 80, Shanghai 200030, China

102 The Observatories of the Carnegie Institution for Science, 813 Santa Barbara St., Pasadena, CA 91101, USA

103 University of Michigan, Ann Arbor, MI 48109

104 Massachusetts Institute of Technology, Cambridge, MA 02139

105 INAF-Italian National Institute of Astrophysics, Italy

106 Space Telescope Science Institute

107 Department of Physics, Duke University, Durham, NC 27708, USA

108 Duke University and Triangle Universitites Nuclear Laboratory, Durham, NC 27708

109 División de Ciencias e Ingenierías, Universidad de Guanajuato, León 37150, México'

110 University of Houston, Houston, TX 77204

111 Syracuse University, Syracuse, NY 13244

112 Max-Planck-Institut für Astrophysik, Karl-Schwarzschild-Str. 1, 85741 Garching, Germany

113 Center for Astrophysics and Cosmology, University of Nova Gorica

114 University of Edinburgh, EH8 9YL Edinburgh, United Kingdom 